den gegebenen Zusammenhängen zwischen. Niere und Gravidität die Niere in zweiter Linie noch mit herangezogen werden, obwohl wir ja auch bei Diabetes minimus als Vorläufer eines echten Diabetes ein ähnliches Verhalten der Niere (Glykosurie ohne abnorm hohe Blutzuckerwerte) kennen.

Zusammenfassung: I. Sämtliche in der Literatur veröffentlichten Untersuchungen einschließlich der vorliegenden haben mit der Frank-NothmaNnschen Schwangersehaftsglykosurieprobe in $85 \%$ positive, in I $_{5} \%$ negative Resultate ergeben. Die Probe ist demnach nur als wahrscheinliches Schwangerschaftszeichen $\mathrm{zu}$ werten.

2. Als Ersatz für den teuren Traubenzucker scheint Kunsthonig verwertbar zu sein.

3. Die Adrenalinglykosurie ist nach vorliegenden Untersuchungen für die Diagnose Schwangerschaft unbrauchbar.

4. Die Schwangerschaftsglykosurie ist auf Grund von Blutzuckerkurven als eine Glykosurie ex hyperglycaemia aufzufassen. Sie ist eine alimentäre Glykosurie bei krankhaft herabgesetzter Assimilationsgrenze für Traubenzucker. Ursache ist eine durch die Schwangerschaftsveränderungen im Körper der Frau (Hypophyse, Schilddrüse, Leber) bedingte Kohlenhydratstoffwechselstörung.

Lit e r a tu r: Bang, Der Blutzucker. J. F. Bergmann, 19r 3. BAvG, Mikromethoden zur Blutuntersachung. J. F. Bergmann, 1920. - Bergsma, Zeitschr. f. Geburțsh. u. Gynäko!. 72. 1912. Brintizer, Inaug.-Diss. Breslau, I922 (Auszug). - BRÖSAMLEN, Dstch. Arch. f. klin. Med. I37, 229. 1921. - Crainiciano u Popper, zit. nach Retzlaff, - Didier u. Philippe, zit. nach RETZLAFF - EISNER u. FORSTER, Berl klin. Wochenschr I92I S. 839. - Eppinger, Falta u. RÜdinger, Zeitschr. f. klin. Med. 66 u. 67. - Eppinger u. Hess, Zeitschr. f. klin. Med. 67. - Frank Arch. f. exp. Pathol. u. Pharmakol. 72, 337. I9I3. - FRANK Kongre $\beta$ f. inn. Med. I9I 3 u. 192 r. - Frank u. Nothmann, Münch. med Wochenschr. I920, S. I 433. - FrankL-HochWART, Die Erkrankungen d. weibl. Genit. usw. I. I9I2. - GotTschalk Klin. Wochenschr. 1922, S. 750, Ref. - GRÜNTHAL, Inaug.-Diss. Breslau 1920. - HoFBAuER, Zentralbl. f. Gynäkol. I922, S. 349 u. I92 I, Nr. 50 und Arch. f. Geburtsh. น. Gynäkol. 93. - JÄGER Zeitschr. f. Geburtsh. u. Gynäkol. 74. I9I3. - KAMNITZER u. JosEpH, Therap. d. Gegenw. 1921, H. 9. - KÜSTNER, Klin. Wo chenschr. I922, Nr. 7. - LEMBCKE u. LiNdig, Monatsschr. f. Geburtsh. u. Gynäkol. 56, S. 285. - LEscrike, Wien. med. Wochenschr. Io2 I, Nr. I. - ManN, Zeitschr. f. klin. Med. 78. igr 3-Merletti Frommels Jahresber. 1905, zit. nach MANN. - NürNBerger, Dtsch. med. Wochenschr. I921, S. 1 I 24. - v. Noorden-SAlomon, Handb. d. Ernährungsl, I. I920. - v. NoordeN-Kaminer, Krankheit. u. Ehe, IJ. Aufl. rgr6. - v. Noorden, Die Zuckerkrankh. und ihre Behandl. 7. Aufl. r9i 7. - Novak, Porges u. Strisower, Dtsch. med. Wochenschr, I9I2, Nr. 40. - Novak u. Porges, Berl. klin. Wochenschr. I9I2, S. 2255 u. I9I I, Nr. 39. - NovaK 1a Frankl-Hochwart. - RetzLAfF, Klin. Wochenschr I922, Nr 7, S. 850. - ROSFNBERG, Klin. Wochenschr. I922, Nr. 8. Roubitschex, Klin. Wochenschr. 1922, Nr. 5. - Schirokauter, Berl. klin. Wochenschr. IgI2, Nr. I I u. 38. - SchmiedeBERG, Arch. f. exp. Terap. u. Pharmakol. 90. I92 : - SEITZ u. JESS, Münch. med. Wochenschr. 1922, Nr. 8. - Straub, Zeitschr. f Hiñ. Mèd. 9r, S. 44. - STolper, Gynäkol. Rundsschau I9r3, Nr. 3. - TACHAu, Arch. f. klin. Med. ro4, 448, I9r r. - Wrdal, zit. nach RETZLAFF.

\section{DER EINFLUSS DES SALVARSANS AUF DIE BILIRUBIN-REAKTION IM BIUTSERUM BEI LUESKRANKEN, ZUGLEICH EIN BEITRAG ZUR FRAGE DER SALVARSANSCHÄDIGUNGEN.}

\section{Strauss und W. Buerkmanis;}

Assistenten der medizin. Univ.-Poliklinik Frankfurt a. M. (Prof. STRASBURGER) und der 2. städt. Abt. für Hant- und Geschlëchtskrankheiten Frankfurt a. M. (Pró́. ALTMANN).

Uberblicken wir die große Literatur auf dem Gebiete der Schädigungen der Leber durch Salvarsan und betrachten wir die diesbezüglichen Ergebnisse der experimentellen Untersuchungen am Menschen und Tier, so erscheint uns eine Wiedergabe oder auch nur kurze Obersicht fast unmöglich.
Die klinischen Beobachtungen und Erfahrungen der Kriegsund Nachkriegsjahre haben zu keiner einheitlichen Auffassung der Leberschädigungen durch Salvarsan geführt, wofür die Sitzung der Berliner Medizinischen Gesellschaft vom 23. I. $1922^{1}$ ) beredtes Zeugnis ablegt. Es würde zu weit führen, an dieser Stelle auf die gesamte Literatur einzugehen. Nur sei uns im Hinblick auf unsere nachfolgenden Untersuchungen gestattet, kurz folgende Tatsachen anzuführen.

Mit B. Fischer ${ }^{2}$ ), G. HerXheImer ${ }^{3}$ ) und BuschkE ${ }^{4}$ ) unterscheiden wir zunächst den Icterus syphiliticus praecox, wie er namentlich im Primär- und Sekundärstadium der Lues auftritt und als zu ihm gehörig mehr oder weniger leicht auftretende funktjonelle Schädigungen der Leber durch die Syphilis selbst oder ihre Toxine. Ferner ist nach den gleichen Autoren durch nichts bewiesen, daß die nach Salvarsanbehandlung bisweilen auftretende akute gelbe Leberatrophie durch das Salvarsan und nicht durch den Lueserreger selbst hervorgerufen wird. Eine Weiterbehandlung mit Salvarsan kann in einzelnen Fällen [UMBER $\left.\left.{ }^{5}\right)\right]$ sogar zur Heilung führen. Aber auch hier steht noch Meinung gegen Meinung. Am ungeklärtesten sind die etwa in der Mitte zwischen diesen beiden Gruppen stehenden Fälle von Ikterus, der gewöhnlich unter dem Bilde des sogenannten Icterus catarrhalis im Verlaufe oder gleich nach Beendigung einer Salvarsankur auftritt, meist mit Magen-Darmstörungen beginnt und zum größten Teil günstig verläuft. REHDER und BECKMANN ${ }^{6}$ ) haben dann als neues Krankheitsbild den Spätikterus nach Salvarsan aufgestellt, der etwa 2 bis 5 Monate nach einer Salvarsankur auftritt, gewöhnlich jedoch 3 Monate nach der letzten Injektion. Auch Zimmern ${ }^{7}$ ) und PuLver$\mathrm{MACHER}^{8}$ ) schließen sich der Abgrenzung dieser Erkrankung an und auch spätere Autoren glauben mit ihnen das Salvarsan in den meisten Fällen als ätiologischen Falktor der Erkrankung bezeichnen zu dürfen.

$O b$, nun die einen Autoren die Lues selbst, die anderen das Salvarsan und hierbei wieder die Höhe der Dosis, die Art des Präparates, die Pausen zwischen den Injektionen und mancherlei andere Umstände für die Schädigungen verantwortlich machen, so sind doch fast alle darin einig; dab die schlechte und namentlich an Eiweiß knappe Ennährung der Kriegszeit die Leber mehr oder weniger funktionell widerstandsunfähig gemacht hat.

Es ist nun verständlich, daß man angesichts der sehr schwierigen klinischen Beurteilung in neuerer Zeit versucht hat, mittels Funktionsprüfungen der Leber Klarheit $z u$ schaffen. Dabei sind wir uns wohl bewußt, daß bei der Vielseitigkeit der Leberfunktionen und der Schwierigkeit ihrer Deutungen Schlüsse auf das pathologische Geschehen nur mit Vorbehalt erlaubt sind.

So hat TACHAU $^{9}$ ) in sehr schönen Untersuchungen durch die Funktionsprüfung der Leber mit Hilfe der Blutzuckeruntersuchung zeigen können, daß beim Icterus syphiliticus praecox eine gewisse Störung im Kohlenhydratstoffwechsel der Leber vorliegt, daß dagegen ein schädlicher EinfluB des Salvarsans auf die Leber bei der üblichen kombinierten $\mathrm{Hg}$-Salvarsankur nicht beobachtet wird. Bekannt ist ferner durch Buschke das Auftreten von Urobilin und Urobilinogen im Urin etwa $6-8$ Stunden nach Salvarsaninjektionen. KIRCH und FREUNDLICH ${ }^{10}$ ) haben dies ebenfalls konstatieren, können. KLEEBERG ${ }^{1 \mathrm{li}}$ ) hat mittels der Galaktoseprobe bei 3 von 2 I Fällen von Lues I und II die vor der Kur positive Probe nach der Salvarsanbehandlung schwinden sehen. Uber Störungen des Eiweißstoffwechsels und ihre Beeinflussung durch Salvarsan ist nichts bekannt. Funktionsprüfungen dieser Art scheinen sich, wie auch solche, die Störungen der entgiftenden Funktion der Leber betreffen, als praktisch verwertbar nicht eingebürgert zu haben. Was die Störungen in der Verarbeitung der Gallenfarbstoffe betrifft, so sind wir gerade in letzter Zeit durch die Untersuchungen EPPINGERs ${ }^{12}$ ), Hidmans VAN DEN BERGH ${ }^{13}$ ), LePEHNES ${ }^{14}$ ), RosenTHALS ${ }^{15}$ ) und ThanNhausers ${ }^{16}$ ) in der Lage, mit sehr verfeinerter Methodik auch nur geringe Spuren von Gallenfarbstoff im Blute nachzuweisen.

Und hièrbei ist es für unsere Frage gleichgültig, ob wir mit EPPINGER und anderen Autoren die Leberzelle als Ausscheidungsorgan des Bilirubins oder mit MINxowsizi ${ }^{1}$ ) Funktionsstörungèn der Léberzelle in bezug auf die Gallebereitung annehmen. Vielmehr nehmen wir mit H. v. D. BERGH ähnlich wie bei manchen Fällen von Herzfehlern und Gallensteinanfällen auch bei unseren Untersuchungen, wie weiter unten ausgeführt sei, eine Schädigung oder Insuffizienz in einer Teilfunktion der Leberzellen an. Denn auch bei 
normaler Zufuhr von Gallenfarbstoff nach den Leberzellen und bei ungehindertem $A b f l u B$ der Galle wird es zu einer Erhöhung des Bilirubingehaltes im Blute kommen können, wenn die Leberzellen in ihrer sezernierenden Funktion gelitten haben. Im allgemeinen scheint eine Beeinträchtigung der sezernierenden Funktion der Leberzellen für Bilirubin und Urobilin das erste Symptom einer Läsion des Leberparenchyms zu sein. Da der Schwellenwert für die Ausscheidung des Urobilins durch die Nieren sehr tief liegt, erscheint das den Leberzellen entschlüpfte und im Blut zurückbleibende Urobilin sofort im Urin. Der Schwellenwert für die Ausscheidung des Bilirubins durch die Nieren liegt aber sehr hoch (etwa bei einer Konzentration von $I: 5^{\circ}$ ooo). Das durch die Leberzellen nicht ausgeschiedene Bilirubin bleibt also im Blutstrom kreisen.

Es würde in diesem Rahmen zu weit führen, auf alle Methoden des Bilirubinnachweises im Blute einzugehen. In Ubereinstimmung mit Holzer und MEHNER ${ }^{18}$ ), die eine gute Kritik fast aller zur Zeit gebräuchlichen Methoden geben, fanden wir die $H$. v. D. BERGHsche Methode für relativ kleine Mengen des Gallenfarbstoffes, wie sie bei unseren Untersuchungen in Betracht kommen, am weitaus geeignetsten.

Die Methode nach Meulengracht, die Wechselmann und HoHORsT ${ }^{19}$ ) zu ähnlichen Untersuchungen benutzten, ist zwar sehr viel einfacher, aber unzureichend. Dies betont ebenfalls ScHNeI$\mathrm{DER}^{20}$ ), dessen Befunde sich in vielem mit den unsrigen, wie unten weiter ausgeführt sei, decken.

Kurz die Methodite unserer Untersuchungen, wobei wir, was Einzelheiten betrifft, auf H. v. D. BrRGHS Monographie ,Der Gallenfarbstoff im Blute" verweisen. In ein Zentrifugenröhrchen bringt man zuerst $0,5 \mathrm{ccm}$ klares Serum, dazu I,0 96 proz. Alkohol, darnach scharf zentrifugieren und von der obenstehenden Flüssigkeit I ccm in das Gläschen des Authenriethschen Colorimeters bringen. Alsdann fügt man 0,25 des stets frisch bereiteten Diazoniumgemisches hinzu, wartet einen Augenblick und liest ab. Da die Flüssigkeit im Gläschen fast stets trübe ist, wird grundsätzlich o,5 Alkohol noch hinzugefügt, was bei der Berechnung berücksichtigt werden muß. Als Vergleichskeil dient ein fertiger Bilirubinkeil. Die Umrechnung machten wir, wie es auch LEPEHNE und BotzIAN $\left.{ }^{21}\right)$ getan haben, der Einfachheit halber in Bilirubineinheiten (B.-E.), wobei z. B.

$$
\begin{aligned}
& \text { 0,5 B.-E. }=0,5 \cdot \frac{\mathrm{I}}{200000}=\frac{\mathrm{I}}{400000}, \\
& \text { I,0 B.-E. }=\mathrm{I} \cdot \frac{\mathrm{I}}{200000}=\frac{\mathrm{I}}{200000}
\end{aligned}
$$

bedeuten.

Natürlich mußten wir zunächst bei einer großen Zahl (insgesamt über 50 Fälle) denBilirubingehalt beim normalenMenschen prüfen und wie es auch ScHNEIDER getan hat, fortlaufende Bestimmungen in bestimmten Abständen beim selben Menschen vornehmen. Das geringe physiologische Schwankungen des Bi-Spiegels beim selben Individuum vorkommen, ist bekannt (LEPEHNE, H. v. D. BERGH, BotzIAN u. a.) jedoch sind diese Schwankungen bei Einhaltung genauester Technik, gleicher Apparatur, sauberem Pipettieren und guter Übung im Colorimetrieren sehr gering. Sie betragen bei unseren Normalfällen ungefähr bis 0,2 und 0,3 B.-E. Die Bilirubinwerte unserer Testfälle bewegen sich zwischen 0,3 B.-E. und I, I B.-E., der Durchschnittswert ist 0,6 B.-E. Es stimmen also unsere Normalwerte mit den Befunden anderer Untersucher $(\mathrm{H}$. v. D. Bergh, Lepenne, Thannhauser, Schneider, gut überein. Fälle mit sogenannter physiologischer Hyperbilirubinämie ( $H$. v. D. BERGH) sind sehr selten und können bei der großen Zahl der ausgeführten Bestimmungen vernachiässigt werden.

Um nun den Einfluß des Salvarsans - verwandt wurde in der weitaus größten Zahl der Fälle Neosilbersalvarsan, in einigen Fällen Neosalvarsan und Salvarsannatrium - auf den Ablauf des Bilirubinspiegels zu prüfen, gingen wir von folgenden Fragestellungen aus:

I. Wie verhält sich der Bilirubinspiegel im Verlauf einer Salvarsankur:

a) bei normalem,

b) bei erhöhtem Bilirubingehalt im Serum bei Beginn der Behandlung?

II. Welchen Einflu $\beta$ hat die einzelne Salvarsangabe auf den Bilirubinspiegel?

III. Wie verhält sich der Bilirubinspiegel 3-5 Monate nach einer Salvarsankur?
An dieser Stelle ist besonders zu vermerken, daß, um ein möglichst genaues Bild der Salvarsanwirkung zu erhalten, Quecksilber nicht gegeben wurde, im Gegensatz zu den Untersuchungen von SCHNEIDER und WECHSELMANN und HOHORST, deren Ergebnissen kombinierte $\mathrm{Hg}$-Salvarsankuren zugrunde lagen.

Bei der I. Gruppe kamen 37 Patienten, meist Mäđchen unđ Frauen mit Lues I und II, in Betracht, da wir wissen, daB gerade in diesen Stadien syphilitische Leberveränderungen (Icterus syphiliticus praecox und verwandte, leichtere Formen) relativ häufig vorkommen. Von diesen 37 Fällen håtten 28 normalen Bilirubingehalt, und zwar von 0,4 bis $\mathrm{r}, 2$ B.-E., im Durchschnitt 0,7 B.-E. als Werte bej Beginn der Behandlung. Gewöhnlich wurden im ganzen $4-5 \mathrm{~g}$ Salvarsan gegeben und in bestimmten Abschnitten während der Kur die Bestimmung wiederholt. Hierbei zeigte sich außer den obenerwähnten physiologischen Schwankungen kein Anund Absteigen des Bilirubinspiegels. Am Ende der Behandlung war fast stets der normale Wert vorhanden, im Durchschnitt 0,7 B.-E.

Bei 9 Fällen dieser Gruppe war bei Beginn der Kur eine deutliche Hyperbilirubinämie als Zeichen einer wahrscheinlich doch luetischen Leberaffektion zu konstatieren. Meist war Urobilinurie, vereinzelt auch eben angedeuteter Subikterus nachweisbar. Leider können wir infolge Raummangels ausführliche Krankengeschichten nicht wiedergeben und nur summarisch berichten. Als typisches Beispiel dieser Untersuchungsserie sei folgender Fall kurvenmäßig dargestellt:

Fr1. H. D., Lues II seropositiva. Urin: Ug positiv. Ganz leichte Leberschwellung. Roseola.

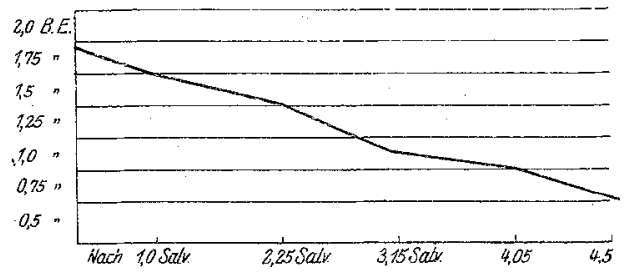

Die Vertikale gibt die Werte fär den Gallenfarbstoff, in B.-E. ausgedrückt, an; auf der Horizontalen sind die Gesamtsalvarsanangaben vermerkt. Von einem Gehalt an 1,85 B.-E. im Serum bei unserer Patientin als deutliches Symptom eines sekundär luetischen Leberinfektes langsames und konstantes Absinken des Bi-Gehaltes unter Neosilbersalvarsanbehandlung bis auf normalen Wert $(0,75$ B.-E.).

Im ganzen betragen die Ausgangswerte unserer 9 Fälle I, 2-2,4 B.-E., im Durchschnitt I,7 B.-E. Die bei diesen Patienten, ähnlich wie im obigen Falle, vorgenommenen BiBestimmungen ergaben bis zum SchluB der Kur Absinken der anfangs erhöhten Werte auf normalen oder fast normalen Bi-Gehalt, und zwar auf durchschnittich o,8 B.-E. Diese Untersuchungen scheinen uns zunächst eine Bestätigung der schon von HerXHEIMER, B. Fischer, BUSCHKE und einer großen Reihe späterer Forscher angenommenen und latent verlaufenden Leberveränderungen bei Lues I und II zu sein, deren günstige Beeinflussung durch Salvarsan sich dem Auge sonst entzieht, jedoch gemessen an dem Bilirubinspiegel im Serum in fortlaufenden Untersuchungen außer Frage steht.

Uns interessierte nun vor allen Dingen der Einfluß der einzelnen Salvarsaninjektion auf den Bilirubinspiegel des Luetikers. Wir gingen so vor, daß wir $0,3,0,45$ und $0,6 \mathrm{~g}$ Salvarsan (Neosilbersalvarsan, Salvarsannatrium und Neosalvarsan) vormittags injizierten, meist ungefähr nach 6 und nach $20-24$ Stunden Blut entnahmen und untersuchten. Selbstverständlich wurde auch vor der Injektion Serum zur Bestimmung entnommen. Bei to von im ganzen $3^{8}$ Fällen dieser Gruppe konnte nun ein deutlicher Anstieg von 0,4 bis 0,9 B.-E., etwa $20-24$ Stunden - manchmal auch früher festgestellt werden. Nach 36 und ${ }_{4}^{8}$ Stunden vorgenommene Untersuchungen ließen meistens wieder den Ausgangswert feststellen. DaB dieser Anstieg über das $\mathrm{MaB}$ der physiologischen Schwankungen hinausgeht, geht aus den obenerwähnten. Kontrollbestimmungen hervor. Bei der Injektion 
von $0,15 \mathrm{~g}$ Salvarsan war diese Reaktion nicht bemerkbar. Ein Unterschied zwischen den Dosierungen von $0,3,0,45$ und $0,6 \mathrm{~g}$ konnte nicht sicher festgestellt werden. Eine besondere Differenz im Präparat (Neosilbersalvarsan, Neosalvarsan oder Salvarsannatrium) war mit absoluter Sicherheit deswegen nicht zu eruieren, weil die Zahl der mit Neosalvarsan und Salvarsannatrium in dieser Weise untersuchten Fälle im Vergleich zu den mit Neosilbersalvarsan behandelten zu einem ganz sicheren Urteil zu klein war. Prozentual ist die Zahl der oben beschriebenen Reaktionen beim Neosilbersalvarsan um ein weniges geringer. Die Erhöhung des Bi-Spiegels traf ferner Fälle mit normalem Bilirubingehalt, wie auch solche mit erhöhter Gallenfarbstoffmenge im Serum. Folgende Kurve illustriert sehr schön den Ablauf dieser Reaktion bei beiden Typen.

Frl. R. Lues II seropositiva. Urin: Ug positiv, I,9 B -E. als Anfangswert.

Herr St. Lues II seropositiva. Urin: Ug $-0,6 \mathrm{~B}$ E. als Anfangswert.

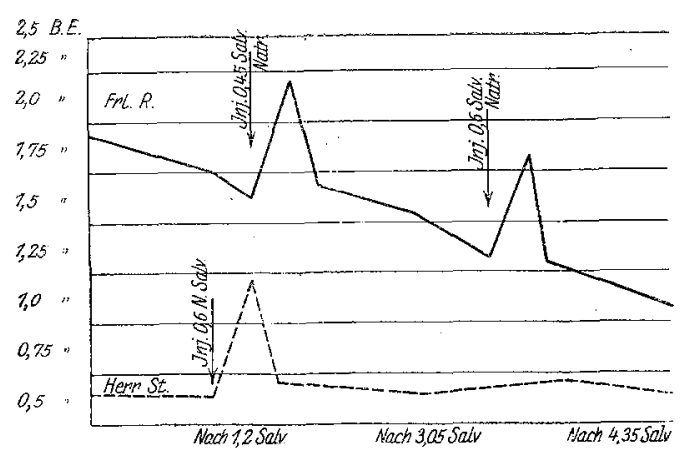

Auch in dieser Kurve sind die Bilirubinwerte in B.-E. ausgedrückt auf der Vertikalen, die Gesamtsalvarsangaben auf der Horizontalen vermerkt. Die einzelnen Salvarsaninjektionen sind besonders mit Pfeilen bezeichnet. Bei der Patientin R. mit einem Anfangswerte von 1,9 B.-E. erfolgt, nachdem der Bilirubinwert ganz wenig bis anf $\mathrm{I}, 65 \mathrm{~B} . \mathrm{E}$. gesunken ist, 22 Stunden nach der Injektion von 0,45 Salvarsannatrium ein Anstieg auf 2,4 B.-E., also um 0,75 B.-E.; darnach wieder schnelles Absinken auf I,7 B.-E.

Bei Herrn St. von einem Normalwert von o,6 B.-E. nach 0,6 g Neosalvarsan ein Anstieg auf I,25 B.-E. und gleichfalls ebenso schnelles Zurückgehen. Trotz dieser einzelnen Erhöhungen des Bi-Spiegels wird, wie wir aus der Kurve sehen, der Bilirubinspiegel im ganzen günstig beeinflußt. Wahrscheinlich sind diese, wenn auch nur in $20 \%$ bei den Injektionen auftretenden Reaktionen auf die gleiche Weise zu erklären, wie das Auftreten von Urobilin im Urin etwa 6 bis 8 Stunden nach der Einspritzung (Buschke), das auch wir in vielen Fällen, oft sogar zugleich mit den oben skizzierten Bilirubinsteigerungen konstatieren konnten. In einem kleinen Teil dieser Fälle war zwar Hyperbilirubinämie, aber keine Urobilinurie nachweisbar. Zudem ist der Nachweis des vermehrten Urobilins und Urobilinogens im Urin sehr stark dem subjektiven Ermessen des jeweiligen Untersuchers anheimgestellt, besonders wenn, wie in diesen Fällen, die Reaktionen oft an der Grenze des Normalen stehen.

Nach alledem scheint die einzelne Salvarsangabe im Sinne H. V. D. BerGHs eine leichte ikterogene Wirkung zu haben als Audsruck einer ganz leichten, schnell abklingenden Funktionsstörung der Leberzelle, die sich vielleicht in einem Falle nur in einer Urobilinurie, im anderen Falle in einer geringen Hyperbilirubinämie, zuweilen auch in beidem kundtut. In sehr vielen Fällen wird sie die Leber gar nicht alterieren oder nur so minimal, daß wir es nicht feststellen können. $\mathrm{Ob}$ durch die Annahme einer Funktionsstörung im Sinne H. v. D. BERGHS die Wirkung der einzelnen Salvarsaninjektion restlos erklärt ist, erscheint uns mit Rücksicht auf Untersuchungen von $\mathrm{S}$. ISAAC und $\mathrm{E}$. ADLER ${ }^{22}$ ) fraglich, die fanden, daß die Umwandlung einer Zuckerart in die andere, z. B. der Lävulose in Glukose, an der Intaktheit der Leberzellen gebunden ist. Selbst die ganz gesunde Leber verwertet rectal applizierte Aminosäuren nicht mehr, wenn sie mit solchen Eiweißbruchstücken plötzlich überflutet wird [E. AdLER $\left.\left.{ }^{23}\right)\right]$. Auch konnte im Laboratorium der hiesigen Medizinischen Universitäts-Poliklinik in nicht veröffentlichten Untersuchungen gezeigt werden, daß die Leberzellen unter gewissen Bedingungen, wenn sie übermäßig für die Verwertung von Dextrose partiell in Anspruch genommen werden, mit Ausscheidung von Urobilin im Harn antworten, daß mit anderen Worten bei Mehrbelastung einer Partialfunktion der Leber eine andere Funktion in Mitleidenschaft geraten kann. Ganz ähnlich würde sich auch in unseren Fällen das Auftreten von Urobilin im Urin und die Vermehrung von Bilirubin im Serum erklären lassen, wenn wir bedenken, daß die Leberzelle von dem injizierten Salvarsan das Arsen abspalten und aufspeichern, und wie wir aus den Untersuchungen PAUL EHRIICHs selbst wissen, gewisse Komplexe des Salvarsans oxydieren und an andere Körper binden und so eliminieren. bzw. entgiften mub.

Im Hinblick auf die eingangs erwähnten Fälle von Spätikterus nach Salvarsan unternahmen wir $4^{8}$ Bilirubinbestimmungen bei Patienten, die 2-6 Monate vorher eine intensive Salvarsanbehandlung durchgemacht hatten. Darunter befanden sich solche, die 4 und mehr Kuren hinter sich hatten, meist kombinierte Hg-Salvarsanbehandlung. Bei fast allen Fällen war normaler Bilirubingehalt im Serum vorhanden und zwar betrugen die Werte 0,3-I,2 B.-E., im Durchschnitt 0,75 B.-E. Nur 3 Patienten hatten mäßig erböhte Bilirubinspiegel und zwar I,4; I,5 und I,75 B.-E. Jedenfalls sind auch hier größere Mengen von Gallenfarbstoff im Serum im Sinne einer Funktionsstörung der Leber nicht vorhanden.

Lite ratur: I) Sitzung d. Berlin. med. Ges, vom. 23. I. I922, Med. Klinik ig22, Nr. 8 u. 9. - $\left.{ }^{2}\right)$ B. Fischer, Dtsch, med. Wochenschr. I9I 5, Nr. 32. - 3) G. HerXheimer, Berl. klin. Wochenschr. I920, S. 37I. - ${ }^{4}$ ) Buschke, Berl. klin. Wochenschr. I920, Nr. 6:BuschKe, in RIECKE, Handbuch der Haut- u. Geschlechtskrankh. rgog, S. 603. - 5) Umber, Verein f. inn. Med. u. Kinderheilk. Berl. klin. Wochenschr. I920, Nr. 6. - 6) ReHder u. BeckMann, Zeitschr. f. klin. Med. 94, H. 3-4. I9I7. - 7) Zrmmern, Dermatol. Zeitschr. 27. I919. - 8) Pulvermacher, Dermatol. Zeitschr. 27. I9I9. - 9) Tachau, Dermatol. Zeitschr. 32, 305. I92x. - 10) KIRCA u. Freundeich, Arch. f. Dermatol. u. Syphilis 136. I921. 11) KLEEberG, Med. Klinik I920, Nr. 45. - 12) EPPINGer, Die hepato-lienalen Erkrankungen. Berlin 1920. - 13) V. D. BERGH, H., Der Gallenfarbstoff im Blut. Leipzig r9r8. - ${ }^{14}$ ) LEPEHNE, Dtsch. Arch. f. klin. Med. I32. I920. - I35*. I92I. - 15) Rosenthal, Arch. f, exp. Pathol. u. Pharmakol. 9r. I92I; u. Dtsch. Arch. f. klin. Med. Ig2I. - 16) Thannhauser u. Andersen, Dtsch. Arch. f. -lin. Med. I37. I92 I. - 17) Minkowski, in Mering, Handb. d. inn. Medizin Bd. I. I9I3. - 18) Holzer u. MEHNer, Klin. Wochenschr. I922, Nr. 2. $-{ }^{19}$ ) WEChSELMANN u. HoHORST, Arch. f. Dermatol. u. Syphilis. I36. I92I. - 20) SCHNeIder, Dermatol. Wochenschr. I922, Nr. Io/II. - 21) BorzIAn, Mitteil. a. d. Grenzg. d. Med. u. Chirurg. 32. I920. - 22 ) IsAac, S. u. B. AdLer, Zeitschr. f. physiol. Chemie. 2I. - ${ }^{23}$ ) AdLER, E., Beiträge zur Frage der Assimilation tief abgebauter Eiweißkörper bei rectaler Einverleibung. Frankfurt a. M. I9I4.

Anmerkung bei der Korrektur: Bei der Kcrrektur ersehen wir aus einem Referat, daß LINSER (Tübingen) (Innerer Kongreß I922. Wiesbaden) gleichfalls nach der Injektion von Salvarsan ein Ansteigen des Bilirubinspegels gefunden hat. Spezfische Behandlung hatte ebenfalls ein Atsinken des Bilirubins im Scrum zur Folge. Wir brauchen nicht zu betonen, da $B$ unsere Untersuchungen gänzlich unabhängig von denen LINSERS erhoben wurden.

\section{DIE „,PROTEOSEN“ IN DER KINDERHEILKUNDE. \\ Von \\ Dr. CARI G. LeO-Wolf, Kind эrarzt, Buffalo, N. Y. U. S. A.}

Ersuchen um Separatabdrücke von seiten deutscher und österreichischer Kollegen bringen es mir zum Bewußtsein, daß der Bericht des Herrn Dr. Hans Holfeld ${ }^{1}$ ) in dieser Wochenschrift den Eindruck erweckt hat, als hätte ich über die allergischen Reaktionen der Kinder auf verschiedenerlei

1) Klin. Wochenschr. Nr. II, S. 53 . 